\title{
Smartphone electrocardiogram monitoring: current perspectives
}

This article was published in the following Dove Press journal:

Advanced Health Care Technologies

\author{
Andrew L Walker' \\ Joseph B Muhlestein² \\ 'University of Utah School of \\ Medicine, Department of Internal \\ Medicine, Salt Lake City, UT, USA; \\ ${ }^{2}$ Intermountain Medical Center Heart \\ Institute, Salt Lake City, UT, USA
}

\begin{abstract}
Three-fourths of Americans now own smartphones. Mobile health applications are becoming increasingly common for the management of various chronic diseases, and have the potential to improve health outcomes. One of these is the sector of smartphone electrocardiogram (ECG) technology. Previously, obtaining an ECG has involved a bulky machine requiring medical training to operate. Most of the smartphone ECG technologies involve a single-lead ECG obtained on a small device that communicates with a smartphone. Many companies offer smartphone ECG technology. The most prevalent and studied of these is the Kardia Mobile device by AliveCor ${ }^{\circledR}$, which has a Food and Drug Administration-approved algorithm for detecting atrial fibrillation (AF). Here, we specifically review smartphone ECG technology including model specifications, cost, and ongoing clinical trials. We also review clinical uses of the technology including screening and monitoring of AF, QT monitoring during initiation of rhythm control medications, and the ability to accurately detect ST-elevation myocardial infarction.
\end{abstract}

Keywords: smartphone, ECG, AliveCor, Kardia Mobile, Cardiac Designs, ECG Check, D-Heart, QardiaCore, EPI Mini, iHealth Rhythm, atrial fibrillation

\section{Introduction}

The twenty-first century has seen the convergence of medicine and technology to bring once dedicated, cumbersome, single-function machines to a mobile, compact, and multifunction platform. There is also increasing pressure for inclusion of self-management and mobile health (mHealth) programs in the monitoring of chronic disease, as they have the potential to improve health outcomes. ${ }^{1}$ One of the best examples of this is the smartphone electrocardiogram (ECG). Invented in 1903 by Willem Einthoven, the first ECG machine used a string galvanometer to measure the small electric currents produced by the human heart and project these onto paper. Much like the first computers, the first ECG machines were large, almost room-sized, instruments. These have evolved into the modern-day ECG machine health care workers are familiar with, usually consisting of a small cart on wheels with a laptop-sized device, screen, keyboard, and printer.

The ECG is an essential medical diagnostic test, and this has led to its being found in the medical office, hospital ward, emergency department, and most places in between. Its application is wide and encompasses everything from screening and monitoring to diagnosing cardiac emergencies. Physicians use ECGs to determine cardiac rhythms, measure intervals, and assess for evidence of ischemia, new and old. Because of the high reliance on ECGs being readily accessible and easy to use, there
Correspondence: Joseph B Muhlestein Intermountain Medical Center Heart Institute, 5 2 I S Cottonwood St, Salt Lake City, UT 84I 2 I, USA

$\mathrm{Tel}+\mathrm{I} 80 \mathrm{I} 5074000$

Fax +80I 5074789

Email jbrent.muhlestein@imail.org 
is a push to make them more compact and more ubiquitous. One piece of technology that already fits this prototype is the smartphone, which we define as a device that performs many of the functions of a computer, typically having a touchscreen interface, Internet access, and an operating system capable of running downloaded applications (apps). As of November $2016,77 \%$ of Americans owned smartphones. ${ }^{2}$ Therefore, it is logical to develop a way for the smartphone to function as an ECG machine.

The earliest evidence of smartphones being used to monitor cardiac activity can be seen in smartphone video plethysmography, where a user places his or her finger over the camera lens and flash simultaneously to obtain a heart rate. This progressed to the development of a single-lead smartphone ECG, approved in 2013. Since then, several other companies have followed suit, developing their own versions of smartphone ECGs. They all operate using a similar principle of a collection device, usually consisting of two electrodes, and an application on a smartphone that processes the collected data. The various company apps then allow for users to see the output immediately and, in some instances, forward the data on to their physician. Because a smartphone ECG is considered a medical device, developers must obtain separate US Food and Drug Administration (FDA) clearance for each make and model of smartphone. There are many ECG devices marketed as mobile or portable. ${ }^{3}$ Here, we specifically review the smartphone ECG platforms available at the time of writing this article, including their function, various diagnostic uses, publications that support their validity, and ongoing clinical trials involving their use.

\section{Current technology Kardia Mobile}

The first and most popular of the smartphone ECG technologies, Kardia Mobile and associated Kardia app (AliveCor ${ }^{\circledR}$, Mountain View, CA, USA) received FDA 510(k) approval in December 2012 and has subsequently been FDA and European Conformity cleared. The device consists of a credit-card sized detector pad with two metal electrode squares (Figure 1). Users commonly place two fingers, index and middle, on each of the electrodes for $30 \mathrm{~s}$. The detector pad can be placed across the chest as well. The detector sends the data using ultrasound to the user's smartphone microphone, where the Kardia app's rendering of the data is displayed as a single ECG lead and is most similar to lead I on a conventional 12-lead ECG. Upon first use of the device, an initial ECG is obtained from the user and sent to a physician through the app. Within $24 \mathrm{~h}$, users receive their first ECG report, including an interpretation of the reading. Subsequent readings can be obtained in the same way and are interpreted instantly by the app via one of the three FDA-cleared algorithms: 1, normal; 2, interference; and 3, atrial fibrillation (AF). The AF algorithm was the first to be cleared, followed by the other two in 2015. To comply with FDA regulations, the company notes that a reading of AF is not diagnostic. If the reading does not fit into any of the three algorithms, it will
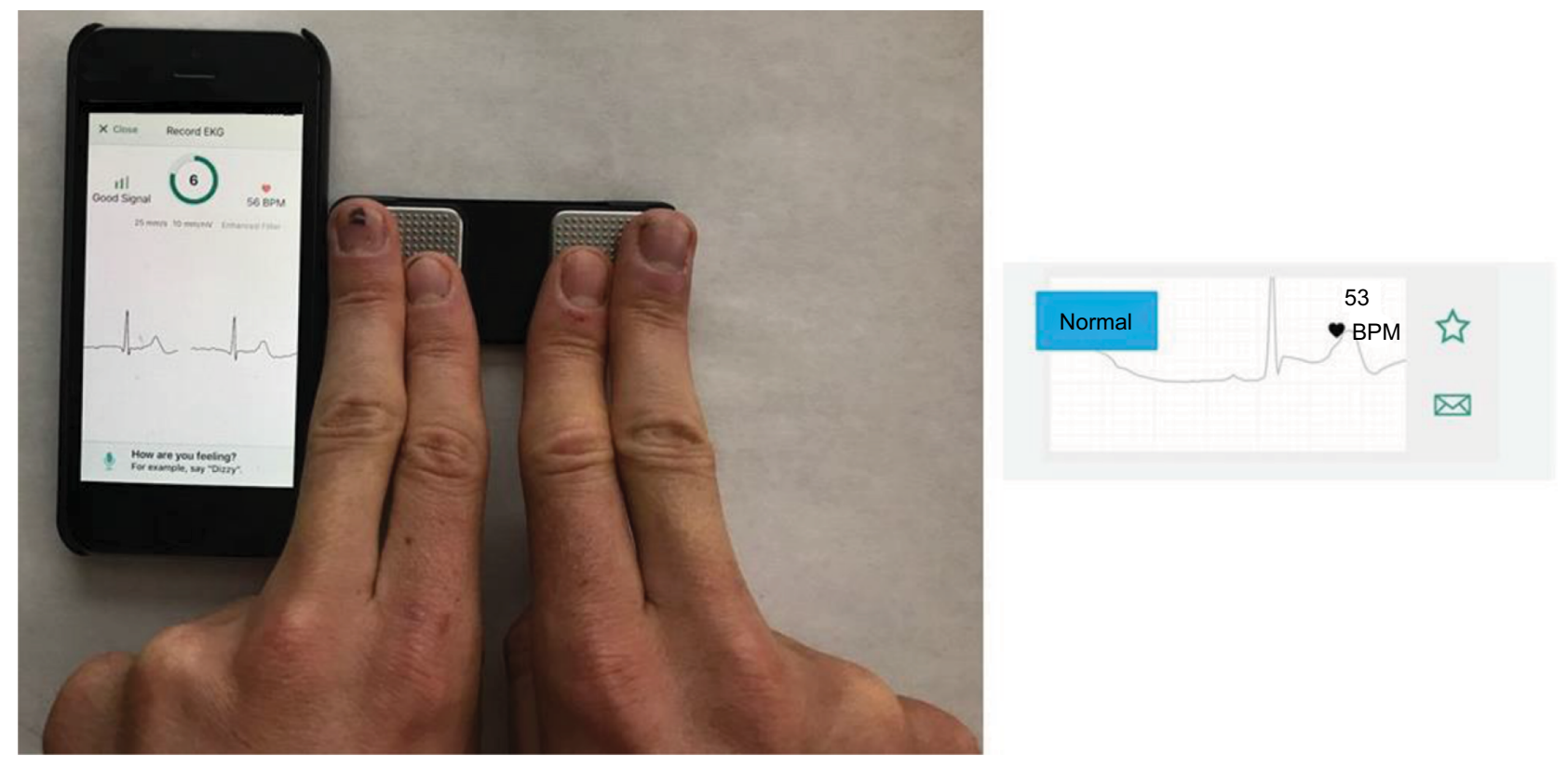

Figure I Kardia Mobile device during recording and interpretation after recording. 
be read as "undetermined." Kardia Mobile is compatible with most Apple and Android smartphone products and currently sells for $\$ 99$ (Table 1). The company notes that the device has not been tested in patients weighing less than $10 \mathrm{~kg}$ or in those with pacemakers or internal cardiac defibrillators.

\section{ECG Check}

In January 2013, just one month following Kardia Mobile's debut, a second and very similar device called ECG Check (Cardiac Designs ${ }^{\circledR}$, Round Rock, TX, USA) was granted FDA 510(k) approval and subsequent FDA clearance. Similar to the previous device, ECG Check consists of a slightly larger detector with two metal electrode squares (Figure 2). A user places a single finger on each of the electrodes for $30 \mathrm{~s}$. The detector then sends the data via Bluetooth to the user's smartphone where the ECG Check app renders the data as a single ECG lead and is most similar to lead I on a conventional 12-lead ECG. What happens next depends on which of the device's two modes, over-the-counter (OTC) or prescription (Rx)/physician mode, is enabled. In OTC mode, users are unable to see the rendered ECG tracing and are only provided with a message stating whether their tracing is normal or abnormal, their heart rate, and the quality of their tracing. In $\mathrm{Rx} /$ physician mode, the same information is provided in addition to the image of the rendered ECG tracing. Users can enable Rx/physician mode either by uploading a prescription for the device from their physician through the app or, if the user is a physician, by entering their National Provider Identifier number and state. Both modes enable users to share their recordings electronically. The company's website indicates that the ability to have a user's tracing reviewed and interpreted by a qualified technician for an additional fee is coming soon. ECG Check is compatible with any iPhone or Android device that supports Bluetooth 4.0 , and comes at a cost of $\$ 139$ (Table 1).

\section{D-Heart}

The D-Heart device (D-Heart ${ }^{\circledR}$, Genova, Italy) comes from a company that places its focus on bringing ECG technology to rural, underserved areas of the world. Because this device is still in the trial phase, full details surrounding its functionality are limited. At the time of writing, what we do know is that it consists of a collection device, similar in size to a conventional round thermostat, with five ECG leads, three peripheral and two precordial. These leads attach to the patient's chest via adhesive electrodes, similar to the ones used by the conventional 12-lead ECG. The data are rendered by the collection device and then streamed via Bluetooth to a smartphone, which displays an eight-lead ECG consisting of leads I, II, III, aVR, aVL, aVF, V2, and V5 in a dedicated smartphone app (Figure 3). This tracing can then be interpreted immediately by a medical professional or it can be transmitted electronically for remote interpretation. The smartphone app incorporates a video guide to aid in appropriate lead placement on the patient. The simultaneous, multi-lead acquisition of this device makes it uniquely different from the single-lead devices above. Early preliminary data showed good concordance between the D-Heart device and conventional 12-lead ECG. ${ }^{4}$ There is ongoing investigation using the device to identify morphological ECG abnormalities in hypertrophic cardiomyopathy patients as well and general ECG screening of pregnant women in rural Africa. ${ }^{5,6}$ The company indicates that the device will have both Apple and Android support. An iPhone 5 is being utilized in the current clinical trials. While the device is not currently on the market, the company reports that its price will be less than $\$ 35$ (Table 1).

\section{QardioCore}

QardioCore (Qardio ${ }^{\circledR}$, San Francisco, CA, USA) is a wearable chest strap that has yet to be approved by the FDA. It generates a single-lead ECG visible on a user's smartphone through the accompanying app via Bluetooth technology. Unlike ECG Check and Kardia Mobile, this app does not provide any interpretation of the data. It does, however, operate in a continuous monitoring mode, rather than generating a $30 \mathrm{~s}$ rhythm strip as with Kardia Mobile and ECG Check. Users can share the data with their medical provider if the provider signs up to receive this information through the company's website. The device is only compatible with Apple products and comes at a cost of $\$ 449$ (Table 1). The device also requires a prescription from a medical professional. On our review, there are no published studies or clinical trials utilizing this specific device. QardioCore is not yet available in the USA.

\section{EPI Mini}

EPI Mini (EPI Mobile Health Solutions, Paragon, Singapore) is a freestanding device consisting of a handheld unit with three sensors which generate a $30 \mathrm{~s}$, single-lead ECG tracing. It received FDA 510(k) premarket clearance in 2013, although it is still not for sale in the USA. Unlike other devices, this device has a display screen on the detector unit to visualize the ECG tracing. This unit can also broadcast the data via Bluetooth to a user's smartphone. In addition, users can pay for a subscription service to have their ECGs interpreted by the company's concierge service and receive 


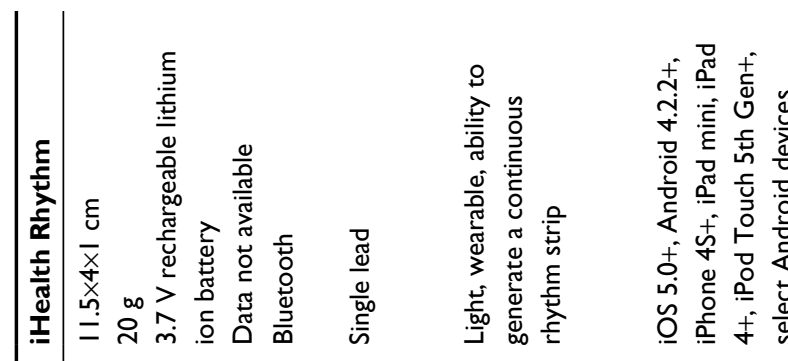

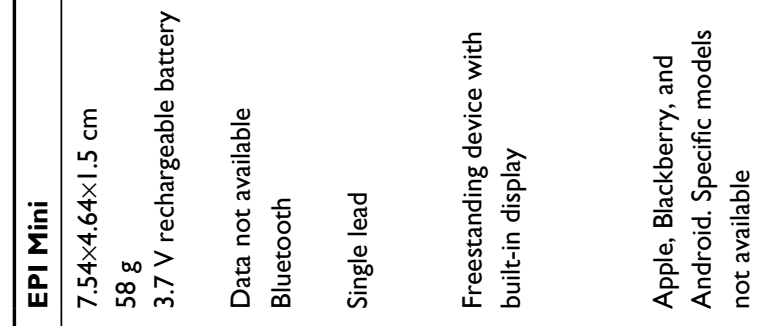

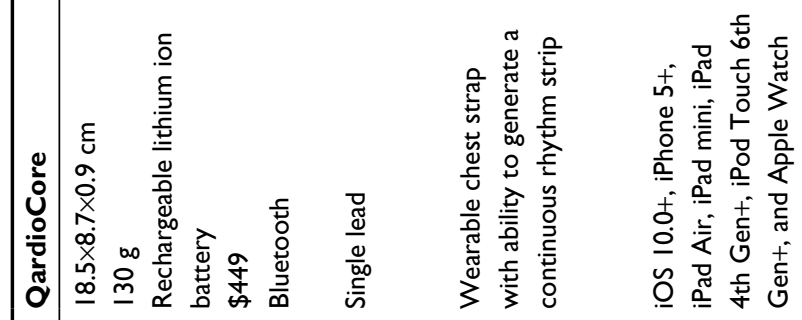

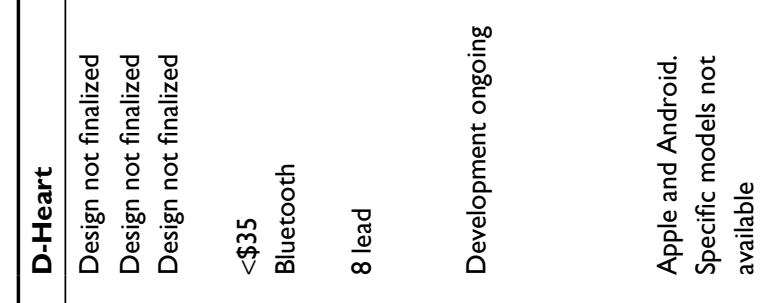

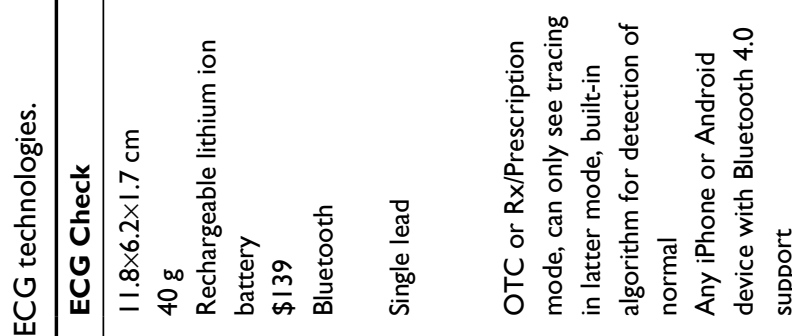

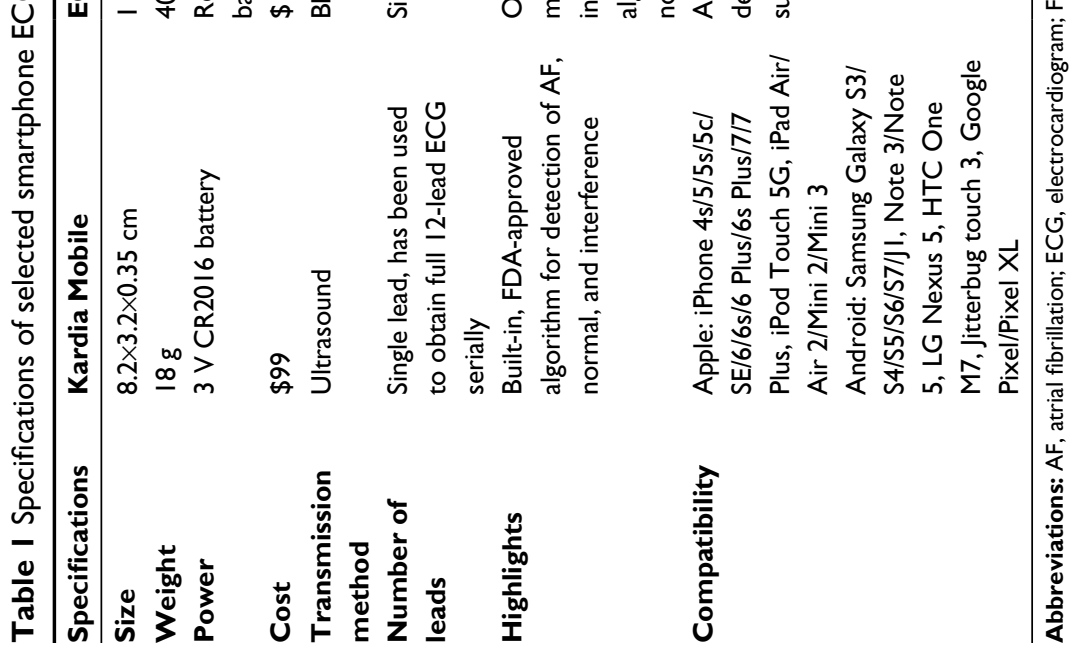




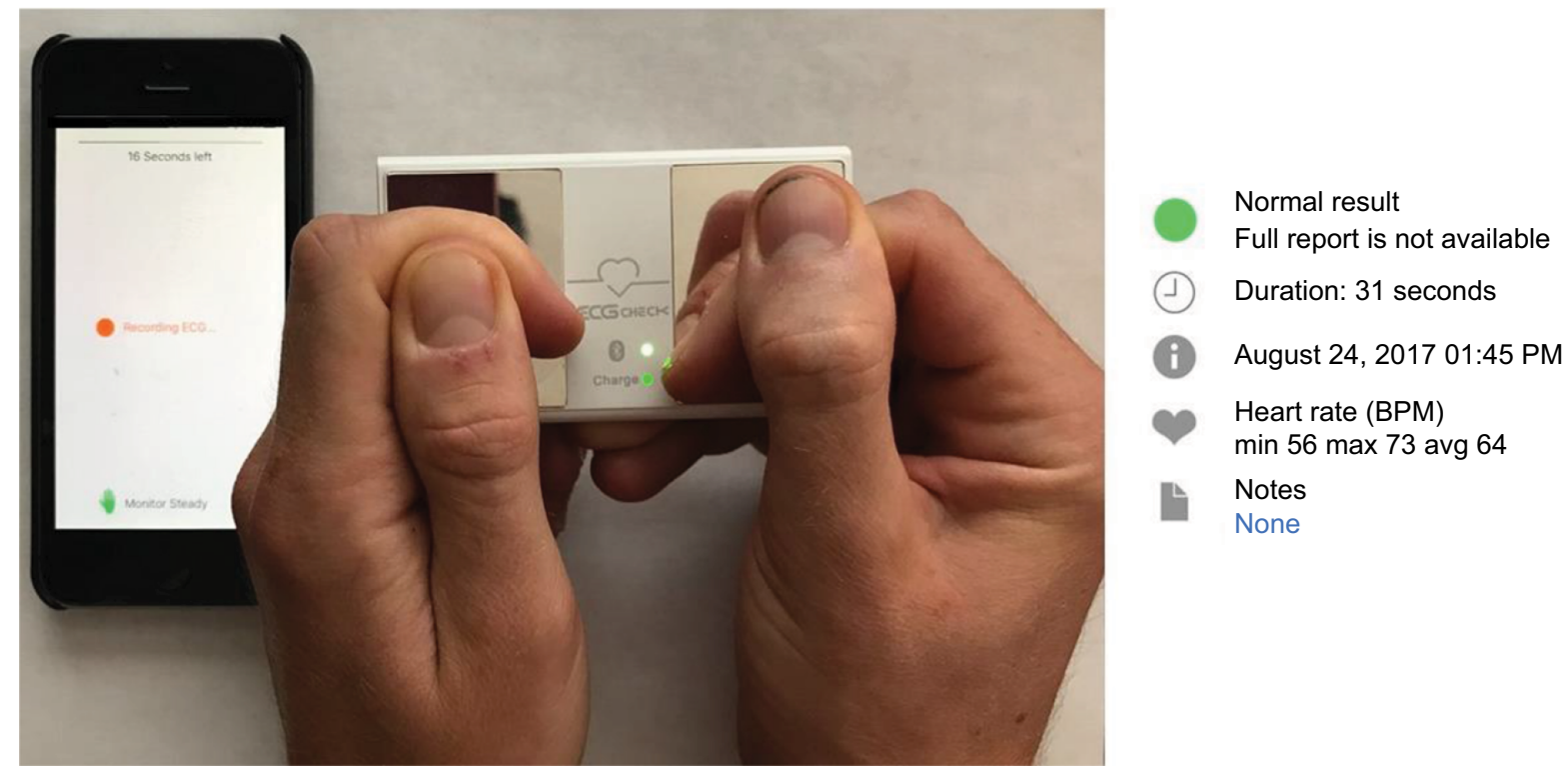

Figure 2 ECG Check device during recording and interpretation after recording.
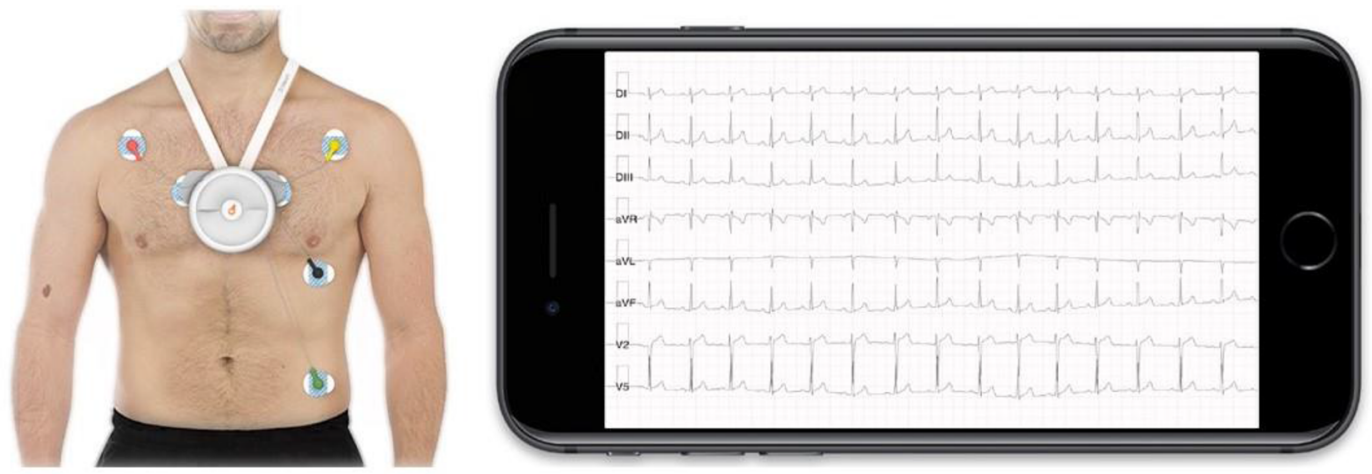

Figure 3 Prototype of D-Heart device lead placement and recording.

a text message with an interpretation. The device requires a prescription from a medical professional. No price is listed on the company's website (Table 1). A predecessor device called EPI Life is a customized mobile phone with the ECG device built into the unit. Because this device is customized and not able to be integrated into consumer-available smartphones, we have elected not to review it further.

In a small, prospective study of 30 healthy individuals, an EPI Mini ECG was compared to an ECG obtained using the company's EPI Life device, which had been previously clinically validated with standard 12-lead. ${ }^{7}$ In this study, each device was used to obtain three single-lead tracings (a modified I, II, V5 tracing) for each participant. The tracings from the EPI Mini correlated with the EPI Life when it came to QRS amplitude as well as PR and QT intervals using regression modeling, with significant accuracy $(p<0.0001) .{ }^{8}$

\section{iHealth Rhythm}

Limited data are available on the iHealth Rhythm (iHealth Labs $^{\circledR}$, Mountain View, CA, USA) as it is yet to be approved in either the USA or Europe. Prototype data from the company's website indicate that the device will be a flat recorder that clips onto a consumable, three-electrode patch. Individuals will be able to monitor themselves for up to $72 \mathrm{~h}$ continuously on the device's internal memory. A single-lead ECG is transmitted via Bluetooth and can be viewed on a smartphone. The device has an event button that the patient can press should they feel any cardiac symptoms, highlighting that time on the ECG. Physicians will have the capability to view the ECG data live via a medical provider app. No price is available on the company's website. The device is reportedly going to support both Apple and Android products (Table 1). 


\section{Utility of smartphone ECG Detection of new AF}

$\mathrm{AF}$ is the most common chronic arrhythmia, with a one-infour risk of lifetime development. ${ }^{9}$ With AF comes increased morbidity and mortality, including a five-fold increased risk of stroke. Most importantly, nearly $20 \%$ of AF-related ischemic strokes occurred in patients with previously undetected AF. ${ }^{10}$ Anticoagulatants can decrease the risk of AF-induced stoke. Despite this and the ability to easily and accurately detect this arrhythmia on routine ECG, universal screening for AF was thought to not be cost effective. ${ }^{11}$

With the development of smartphone ECG technology, this may change. Older, conventional 12-lead ECGs can be cumbersome and require time and knowledge to perform and interpret. With smartphone ECG, a single-lead tracing can be obtained and interpreted in less than 1 min. One device in particular, Kardia Mobile, has an FDA-patented AF detector algorithm, with $97 \%$ sensitivity and $98 \%$ specificity compared to conventional 12-lead ECGs. ${ }^{12}$ Several studies have looked at the possibility of using the Kardia Mobile device for AF screening in the clinic. In one feasibility study, five general medicine clinics in Sydney, Australia, utilized the Kardia Mobile device to screen patients greater than or equal to 65 years old who presented for influenza vaccination. Screening was performed by nurses. Results showed newly identified AF in $0.8 \%$ (8/973) of patients. ${ }^{13}$ In another larger feasibility study conducted in Hong Kong, 13,122 patients underwent community screening using the Kardia Mobile device. The rate of previously undiagnosed AF was $0.8 \%(101 / 13122) .{ }^{14}$ In a third study, smartphone ECG screening for AF was found to be both feasible and cost effective. In the SEARCH-AF study, 10 pharmacies in Australia performed community screening for AF. After exclusions, the prevalence of AF identified by screening was $6.7 \%(67 / 1000)$, and newly identified AF was found in 1.5\% (95\% CI 0.8-2.5) of patients. Assuming an adherence of 55\% to warfarin prescription, the incremental cost-effectiveness ratio of implementing smartphone ECG screening into the community was $\$ 4066$ per quality-adjusted life-year gained and $\$ 20,695$ for preventing one stroke. ${ }^{15}$

Kardia Mobile is the only smartphone ECG on the market that has a built-in AF detector and the only one in our review of published studies utilizing it for screening. ${ }^{12}$ While ECG Check provides an interpretation of normal or abnormal in both of its functionality modes, no further rhythm specification is made beyond that. All devices described above are more portable than conventional ECG machines and could be used to screen for AF as long as they utilized physician interpretation. Aside from their use as screening tools in the health care systems, smartphone ECG devices can be purchased by health-conscious patients to perform home monitoring for AF. Because of the built-in AF detection algorithm, patients may prefer the Kardia Mobile device. If a different device is used, patients can still obtain tracings when they feel they are experiencing AF and have these tracings reviewed by their physician. In addition, some devices offer a paid, third party interpretation service. It is important to note that while AF can be highly suspected on a smartphone ECG tracing, this does not replace the official diagnosis made on a conventional 12-lead ECG in conjunction with clinician interpretation and examination.

Another common, portable method used to screen for AF is a wearable patch electrode such as the Zio XT (iRhythm, San Francisco, CA, USA). Users wear the patch for up to 14 days, during which time the device collects single-lead ECG data. Once the collection period has ended, the device is mailed back to the company and the ordering physician receives a report with interpretation. To our knowledge, there is no published study that directly compares this "spot" or smartphone ECG approach to a patch in regard to screening for AF. There is an ongoing clinical trial called MOBILE-AF (ClinicalTrials.gov, ID: NCT02507986) that could provide some insight into the superior screening method. The study will randomize 200 patients with cryptogenic stroke or transient ischemic attack to either a 7 day Holter monitor or twice daily, 30 s ECG readings with the Kardia Mobile device plus additional readings during cardiac symptoms for a total of 1 year. The primary outcome will be the percentage of patients identified as having new AF. ${ }^{16}$ The first data are expected in 2019. The ultimate application of this clinical trial's results will rest on patient adherence to twice-daily smartphone ECG monitoring for an entire year.

One study that might model what researchers are likely to see in this clinical trial is a study comparing a single-lead, mobile ECG device called Zenicor ECG ${ }^{\circledR}$ (Zenicor Medical Systems, Stockholm, Sweden) to a conventional $24 \mathrm{~h}$ Holter monitor for arrhythmia detection. Like other aforementioned smartphone devices, the Zenicor ECG obtains a "spot" singlelead, $30 \mathrm{~s}$ ECG reading when prompted by the individual. The device has a built-in subscriber identity module (SIM) card which sends the data wirelessly to the company for processing. It does not have smartphone integration, and for this reason was not reviewed further. In this study, 108 consecutive patients referred for $24 \mathrm{~h}$ Holter monitoring for ambiguous palpitations, dizziness, or presyncope were asked to complete a conventional $24 \mathrm{~h}$ Holter monitor and 28 days of twice-daily, $30 \mathrm{~s}$ ECG collections plus additional 
collections when experiencing cardiac symptoms. After exclusions for known arrhythmia and syncope, 95 patients completed the required monitoring. Clinical significant arrhythmias identified included AF, atrioventricular block, and paroxysmal supraventricular tachycardia. The Holter monitor was able to identify three such patients $(3.2 \%, 95 \%$ CI 1.1-8.9) while the Zenicor device identified 12 patients (13.7\%, 95\% CI 8.2-22.0) ( $p=0.0094) .{ }^{17}$ In this case, spot monitoring with a mobile ECG device for 28 days appeared to be more sensitive in detecting arrhythmia, including AF, than a $24 \mathrm{~h}$ Holter monitor.

\section{Monitoring of known AF}

For patients with known AF, there are many instances where monitoring for its presence is useful. Radiofrequency ablation is a common treatment for AF. It is estimated that AF recurrence is $50 \%$ in the first year following treatments, including ablation, that restore normal sinus rhythm. ${ }^{18}$ ECG monitoring after ablation plays an important role in assessing the success of the procedure and guiding management decisions. ${ }^{19,20}$ In the iTransmit study, a small ( $n=60)$, single-center trial comparing conventional transtelephonic monitoring to the Kardia Mobile device for monitoring patients for arrhythmia recurrence after the ablation procedure, the Kardia Mobile device had 100\% sensitivity and $97 \%$ specificity in the detection of AF and atrial flutter. Furthermore, $92 \%$ of patients preferred the Kardia Mobile device over conventional transtelephonic monitoring. ${ }^{19}$

Other patients are placed on rhythm control medications to manage AF. Some of these, such as dofetilide and sotalol, require inpatient monitoring of the QT interval during initiation. It was demonstrated that the Kardia Mobile device could be used to accurately measure the QT interval when compared to conventional ECG. ${ }^{21}$ In a subsequent study $(n=25)$ of patients admitted for dofetilide or sotalol load, a modified Kardia Mobile device with longer electrodes allowing for both a lead I and lead II tracing was utilized. The device was able to detect QTc greater than $500 \mathrm{~ms}$ with 97\% specificity compared to conventional 12-lead ECG. ${ }^{22}$ This suggests the possibility that in the future patients could be initiated on these drugs as well as follow the QT interval every 3 months, as per the recommendation for these drugs, all from home.

In addition to monitoring AF burden postablation and QT interval monitoring in antiarrhythmic drug therapy, the Kardia Mobile device is being utilized in the ongoing iHEART clinical trial (Clinicaltrials.gov ID: NCT02731326). This is a randomized controlled trial to determine whether AF patients who utilize the Kardia Mobile smartphone ECG have greater improvement in markers such as blood pressure and glucose levels as well as improved AF awareness compared to patients who receive usual care defined by published guidelines..$^{20,23}$ If positive, this would be the first trial showing improved health measures when ECG smartphone technology is utilized.

\section{ST-elevation myocardial infarction diagnosis}

In 2016, it was estimated that approximately 660,000 Americans would have a new coronary event and another 305,000 would have a recurrent event. ${ }^{24}$ The most serious of these events, aside from sudden cardiac death, is the ST-elevation myocardial infarction (STEMI). A STEMI requires urgent revascularization and is largely diagnosed on ECG. Obtaining an ECG earlier in STEMI presentation reduces the time to diagnosis and subsequent intervention. In an adapted version of the Kardia Mobile device consisting of two wire leads, 12-lead ECGs can be reconstructed by obtaining serial lead measurements (Figures 4 and 5). In a pilot study of the ongoing ST LEUIS trial (Clinicaltrials.gov ID: NCT02498405), the authors showed that these reconstructed 12-lead ECGs from the Kardia Mobile device demonstrated excellent concordance with standard 12-lead ECGs for the detection of STEMI as well as non-STEMI. ${ }^{25}$ The implication of this study is that a low-cost, compact, and ubiquitous method exists to diagnose STEMI and improve

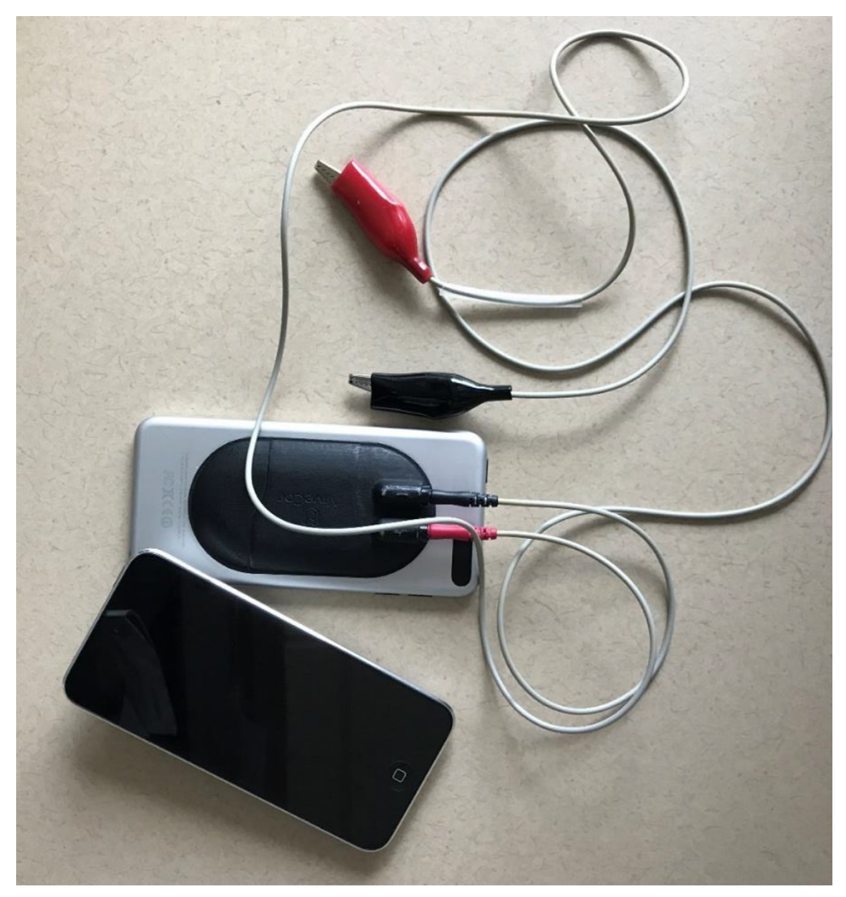

Figure 4 Modified Kardia Mobile device used to reconstruct 12-lead electrocardiograms to detect ST-elevation myocardial infarction.

Note: Reprinted from J Electrocardiol, 48(2), Muhlestein JB, Le V, Albert D, et al, Smartphone ECG for evaluation of STEMI: results of the ST LEUIS Pilot Study, 249259, Copyright 2015, with permission from Elsevier. ${ }^{25}$ 


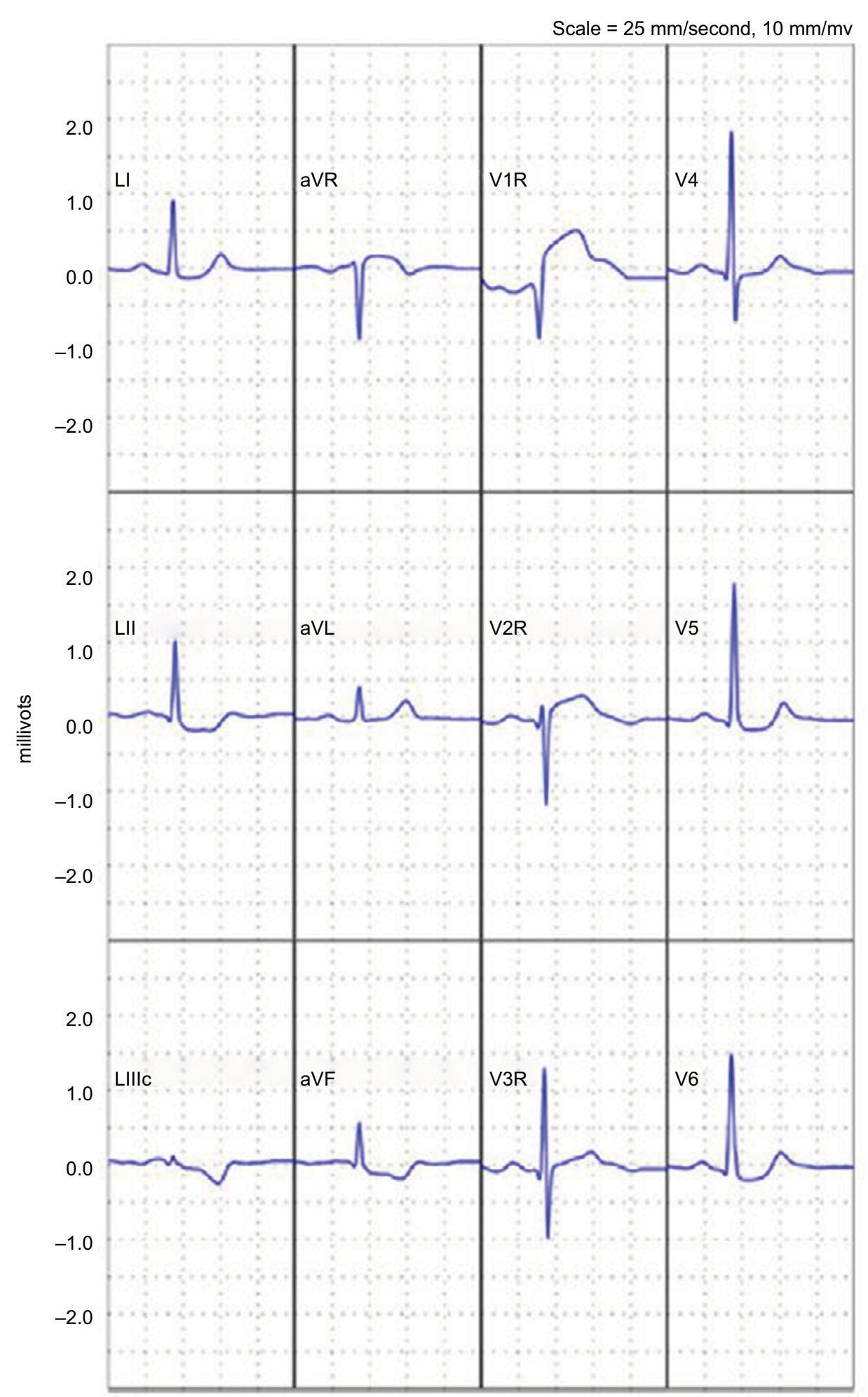

Figure 5 Reconstructed I2-lead electrocardiogram demonstrating an ST-elevation myocardial infarction.

Note: Reprinted from J Electrocardiol, 48(2), Muhlestein JB, Le V, Albert D, et al, Smartphone ECG for evaluation of STEMI: results of the ST LEUIS Pilot Study, 249-259, Copyright 2015, with permission from Elsevier. ${ }^{25}$

time to recognition compared to conventional, 12-lead ECG technology. This method could be utilized by at-risk individuals at home and/or first responders. The authors caution that the data are limited, and this method does not replace STEMI diagnosis with a conventional 12-lead ECG.

\section{Conclusion}

Smartphone ECG technology is a novel, portable, affordable, potentially ubiquitous, and easy-to-use technology with widespread applications for both patients and practitioners. It is an example of mHealth and has the potential to 
improve health outcomes among those living with chronic diseases, through enhanced symptom awareness and control. Here, we have reviewed its use in screening for AF and monitoring of known AF. Health care institutions and health-conscious individuals can utilize the Kardia Mobile's built-in AF detector to reliably detect the arrhythmia or wirelessly transmit tracings obtained on other devices for interpretation by a physician. Smartphone ECG technology can also be used to reliably measure QT intervals in patients taking rhythm-control medications and this raises the question of whether this currently labor-intensive process could be done remotely, even in a patient's own home. Lastly, we reviewed a pilot study that utilized smartphone ECGs to accurately detect STEMI. In the future, this could open doors for home monitoring of high-risk individuals and/ or detect STEMI earlier through increased accessibility to ECG technology.

\section{Disclosure}

The authors report no conflicts of interest in this work.

\section{References}

1. Whitehead L, Seaton P. The effectiveness of self-management mobile phone and tablet apps in long-term condition management: A systematic review. J Med Internet Res. 2016;18(5):e97.

2. Pew Research Center. Mobile Fact Sheet; 2017. Available from: http:// www.pewinternet.org/fact-sheet/mobile/. Accessed August 30, 2017.

3. Guzik P, Malik M. ECG by mobile technologies. J Electrocardiol. 2016;49(6):894-901.

4. Maurizi N, Faragli A, Imberti J, et al. Cardiovascular screening in lowincome settings using a novel 4-lead smartphone-based electrocardiograph (D-Heart ${ }^{\circledR)}$ ). Int J Cardiol. 2017;236:249-252.

5. N Maurizi, C Fumagalli, M Targetti, et al. 217 Comparative analysis of multiple leads smartphone electrocardiograph versus standard 12-leads electrocardiograph in patients with hypertrophic cardiomyopathy. $E P$ Europace. 2018;20(suppl_1):i26.

6. Karijo E. Testing low-cost portable smartphone electrocardiographs in the screening of pregnant women in Mwingi West and Mwingi Central, Kitui County, Kenya; 2016. Available from: https://docs.wixstatic.com/ ugd/5cb79f_27fe047906794f2dbe5a0c500defb369.pdf

7. Lim MCL, Lim I, Devaraj LM. Qualitative and quantitative accuracy of a novel multi-lead mobile phone ECG. Eur Heart $J$ Suppl. 2010;12(suppl_A):S26.

8. Lim M, Lin Z, Michael L. ASSA13-07-1 Comparison of the ECG recordings using a novel mobile ECG recorder (EPI Mini) with a clinically validated mobile phone with ECG recording function (EPI Life). Presented at: Annual Scientific Sessions of APHA. Singapore; 2013. Available from: http://epimhealth.com/images/pdf/AbstractASSA13-07-1.pdf. Accessed January 16, 2018.

9. Lloyd-Jones DM, Wang TJ, Leip EP, et al. Lifetime risk for development of atrial fibrillation: the Framingham Heart Study. Circulation. 2004;110(9):1042-1046.
10. Borowsky LH, Regan S, Chang Y, Ayres A, Greenberg SM, Singer DE. First Diagnosis of Atrial Fibrillation at the Time of Stroke. Cerebrovasc Dis. 2017;43(3-4):192-199.

11. Hobbs FD, Fitzmaurice DA, Mant J, et al. A randomised controlled trial and cost-effectiveness study of systematic screening (targeted and total population screening) versus routine practice for the detection of atrial fibrillation in people aged 65 and over. The SAFE study. Health Technol Assess. 2005;9(40):iii-iv, ix-x, 1-74.

12. Lau JK, Lowres N, Neubeck L, et al. iPhone ECG application for community screening to detect silent atrial fibrillation: a novel technology to prevent stroke. Int J Cardiol. 2013;165(1):193-194.

13. Orchard J, Lowres N, Freedman SB, et al. Screening for atrial fibrillation during influenza vaccinations by primary care nurses using a smartphone electrocardiograph (iECG): A feasibility study. Eur J Prev Cardiol. 2016;23(2 suppl):13-20.

14. Chan N-Y, Choy C-C. Screening for atrial fibrillation in 13122 Hong Kong citizens with smartphone electrocardiogram. Heart. 2017;103(1): 24-31.

15. Lowres N, Neubeck L, Salkeld G, et al. Feasibility and cost-effectiveness of stroke prevention through community screening for atrial fibrillation using iPhone ECG in pharmacies. The SEARCH-AF study. Thromb Haemost. 2014;111(6):1167-1175.

16. Treskes RW, Gielen W, Wermer MJ, et al. Mobile phones in cryptogenic strOke patients Bringing sIngle Lead ECGs for Atrial Fibrillation detection (MOBILE-AF): study protocol for a randomised controlled trial. Trials. 2017;18(1):402.

17. Hendrikx T, Rosenqvist M, Wester P, Sandström H, Hörnsten R. Intermittent short ECG recording is more effective than 24-hour Holter ECG in detection of arrhythmias. BMC Cardiovasc Disord. 2014;14:41.

18. Fetsch T, Bauer P, Engberding R, et al. Prevention of atrial fibrillation after cardioversion: results of the PAFAC trial. Eur Heart J. 2004;25(16): 1385-1394.

19. Tarakji KG, Wazni OM, Callahan T, et al. Using a novel wireless system for monitoring patients after the atrial fibrillation ablation procedure: the iTransmit study. Heart Rhythm. 2015;12(3):554-559.

20. January CT, Wann LS, Alpert JS, et al. 2014 AHA/ACC/HRS guideline for the management of patients with atrial fibrillation: executive summary: a report of the American College of Cardiology/American Heart Association Task Force on practice guidelines and the Heart Rhythm Society. Circulation. 2014;130(23):2071-2104.

21. Chung EH, Guise KD. QTC intervals can be assessed with the AliveCor heart monitor in patients on dofetilide for atrial fibrillation. $J$ Electrocardiol. 2015;48(1):8-9.

22. Garabelli P, Stavrakis S, Albert M, et al. Comparison of QT Interval Readings in Normal Sinus Rhythm Between a Smartphone Heart Monitor and a 12-Lead ECG for Healthy Volunteers and Inpatients Receiving Sotalol or Dofetilide. J Cardiovasc Electrophysiol. 2016;27(7): $827-832$.

23. Hickey KT, Hauser NR, Valente LE, et al. A single-center randomized, controlled trial investigating the efficacy of a mHealth ECG technology intervention to improve the detection of atrial fibrillation: the iHEART study protocol. BMC Cardiovasc Disord. 2016;16:152.

24. Mozaffarian D, Benjamin EJ, Go AS, et al. Heart Disease and Stroke Statistics-2016 Update: A Report from the American Heart Association. Circulation. 2016;133(4):e38-e360.

25. Muhlestein JB, Le V, Albert D, et al. Smartphone ECG for evaluation of STEMI: results of the ST LEUIS Pilot Study. J Electrocardiol. 2015;48(2):249-259. 


\section{Publish your work in this journal}

Advanced Health Care Technologies is an international, peer reviewed, open access journal that provides a unique forum for articles on: point-of-care, health care diagnostics and treatment, bioengineering, biotechnology, biosensing, electronics, clinical/medical science, chemical engineering, materials science, regenerative medicine, micro-/ nano-technologies, and methods and applications for nanoscience and nanotechnology. The manuscript management system is completely online and includes a very quick and fair peer review system, which is all easy to use. Visit http://www.dovepress.com/testimonials.php to read real quotes from published authors.

Submit your manuscript here: https://www.dovepress.com/advanced-health-care-technologies-journal 